\title{
Chiral geometry of higher excited bands in triaxial nuclei with particle-hole configuration
}

\author{
Q. B. Chen and J. M. Yad* \\ School of Physical Science and Technology, Southwest University, Chongqing 400715, China \\ S. Q. Zhang \\ State Key Laboratory of Nuclear Physics and Technology, \\ School of Physics, Peking University, Beijing 100871, China \\ B. Qi \\ Shandong Provincial Key Laboratory of Optical Astronomy and Solar-Terrestrial Environment, \\ School of Space Science and Physics, Shandong University at Weihai, Weihai 264209, China
}

(Dated: June 15, 2018)

\begin{abstract}
The lowest six rotational bands have been studied in the particle-rotor model with the particlehole configuration $\pi h_{11 / 2}^{1} \otimes \nu h_{11 / 2}^{-1}$ and different triaxiality parameter $\gamma$. Both constant and spindependent variable moments of inertial (CMI and VMI) are introduced. The energy spectra, electromagnetic transition probabilities, angular momentum components and $K$-distribution have been examined. It is shown that, besides the band 1 and band 2, the predicted band 3 and band 4 in the calculations of both CMI and VMI for atomic nuclei with $\gamma=30^{\circ}$ could be interpreted as chiral doublet bands.
\end{abstract}

PACS numbers: 21.60.Ev, 21.10.Re, 23.20.Lv

Chirality is a subject of general interest in molecular physics, elementary particle physics, and optical physics. In atomic nuclear physics, the occurrence of chirality was originally suggested in 1997 by Frauendorf and Meng in the particle-rotor model (PRM) and tilted axis cranking (TAC) approach for triaxially deformed nuclei [1]. The predicted patterns of spectra exhibiting chirality were experimentally observed in 2001 [2]. Since then, the investigation of chiral symmetry in atomic nuclei has become one of the most hot topics in nuclear physics. Hitherto, more than 20 candidate chiral doublet bands in oddodd nuclei are proposed in the $A \sim 100, A \sim 130$, and $A \sim 190$ mass regions. In addition, a few more candidates with more than one valence particle and hole were also reported in odd- $A$ and even-even nuclei. For a review, see e.g. [3]. Even though there are many candidate chiral nuclei, the interpretation of the observed pair of near degenerate $\Delta I=1$ bands with the same parity as the chiral doublet bands is still an open question, accompanied with several competitive mechanisms [4].

On the theoretical side, chiral doublet bands were first predicted by particle-rotor model (PRM) and tilted axis cranking (TAC) model for triaxially deformed nuclei [1]. Later on, numerous efforts have been devoted to the development of TAC methods [5 7] and PRM models [8 11] to describe chiral rotation in atomic nuclei. The characters of chiral doublet bands have been examined 12 16]. Recently, the PRM has been extended to the case of many particles and/or holes couples to a triaxially deformed core 17] that allows for the study of more general

*Electronic address: jmyao@swu.edu.cn chiral rotating nuclei. As the counterpart, the interacting boson-fermion-fermion model has also been used to study the chiral doublet bands [18, 19].

In Ref. [20], the possible existence of multi-chiral doublet bands $(\mathrm{M} \chi \mathrm{D})$ in a single-nucleus ${ }^{106} \mathrm{Rh}$ has been proposed based on the triaxial relativistic mean-field calculations and been confirmed later in the similar calculations but with time-odd components 21]. Several minima with large triaxial deformation but with different high- $j$ proton-particle and neutron-hole configurations, which are favorable for nuclear chirality, were found in the calculated potential energy surfaces of the rhodium isotopes ${ }^{104,106,108,110} \mathrm{Rh}[22$.

In comparison with the $\mathrm{M} \chi \mathrm{D}$ that differ from each other in the triaxial deformations and multiparticle configurations, more than one pair of chiral doublet bands may also exist in a single nucleus with the same particlehole configuration, i.e., not only the yrast and yrare bands but also two higher excited bands might be chiral partners. In the PRM with either a rigid or a soft triaxial core, the properties of chiral bands, including the yrast and yrare bands as well as the higher excited bands in an odd-odd nucleus were calculated [23]. It has been shown that the properties of the two higher excited bands (bands 3 and 4), including the reduced probabilities of E2 and M1 transitions between two states with $\Delta I=1$ and staggering patterns, are similar to those of the yrast and yrare bands. It gives us a hint that bands 3 and 4 could be chiral partners as well 23$]$. These facts motivate us to make more detailed theoretical studies for the higher excited bands of chiral rotating nuclei. Particular attention will be paid to examining their chiral geometry.

The PRM, in which the total angular momentum is 


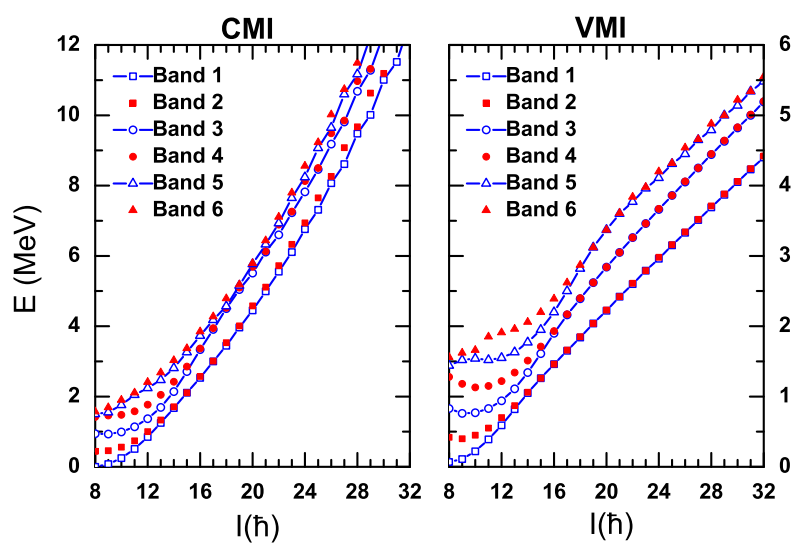

FIG. 1: The six lowest energy levels as a function of spin, calculated by the PRM with the CMI (left panel) and VMI (right panel). The corresponding grouped bands are labeled as band 1-6 in accordance with the increasing energy of the state $8_{\alpha}^{+}$.

a good quantum number, has made great success in the investigation of chiral rotating nuclei in different mass region. Therefore, in this brief report, we would like to adopt this model to make a further study for the lowest six rotational bands (bands 1-6), searching for the higher chiral doublet bands.

The detailed formalism for PRM can be found in Refs. 1, 9, 10]. In the calculations, we take the symmetric particle-hole configuration $\pi h_{11 / 2} \otimes \nu h_{11 / 2}^{-1}$ corresponding to the $A \sim 130$ mass region and the triaxial deformation parameter $\gamma=30^{\circ}$. In the calculation of electromagnetic transitions, the intrinsic quadrupole moment $Q_{0}=(3 / \sqrt{5 \pi}) R_{0}^{2} Z \beta$ is taken to be 3.5 eb. The gyromagnetic ratios $g_{R}=Z / A=0.44$, and $g_{p}=1.21$, $g_{n}=-0.21$ are adopted as Ref. [11]. Both constant $\mathcal{J}_{0}$ (CMI) and spin-dependent variable $\mathcal{J}(I)$ (VMI) moment of inertia are introduced to simulate the rigid and variable cores, where the VMI 24] is taken the same form as Refs. [25, 26]

$$
\mathcal{J}=\mathcal{J}_{0} \sqrt{1+b I(I+1)}
$$

with $\mathcal{J}_{0}=30 \mathrm{MeV}^{-1} \hbar^{2}$ and $b=0.01$.

Figure 1displays the six lowest energy levels $I_{\alpha=1,6}$ as a function of spin $I$, calculated by the PRM with both CMI and VMI. All the calculated levels have been grouped into six bands which are labeled as bands 1-6 in accordance with the order of the energies of the states $8_{\alpha}^{+}$.

As shown in Fig. 1 the degeneracy is found not only between band 1 and band 2, but also between band 3 and band 4 in some spin region. Quantitatively, the energy difference between the band 3 and band 4 in $I \geq 16 \hbar$ region is within $310 \mathrm{keV}$ in the calculation with CMI, and $30 \mathrm{keV}$ in the calculation with VMI. Similar degeneracy with relative larger energy difference is observed between band 5 and band 6 . It is noted that the excitation energy $E(I)$ from the calculation of VMI becomes approximately linear spin-dependent as the spin $I \gg 10 \hbar$, in which case,
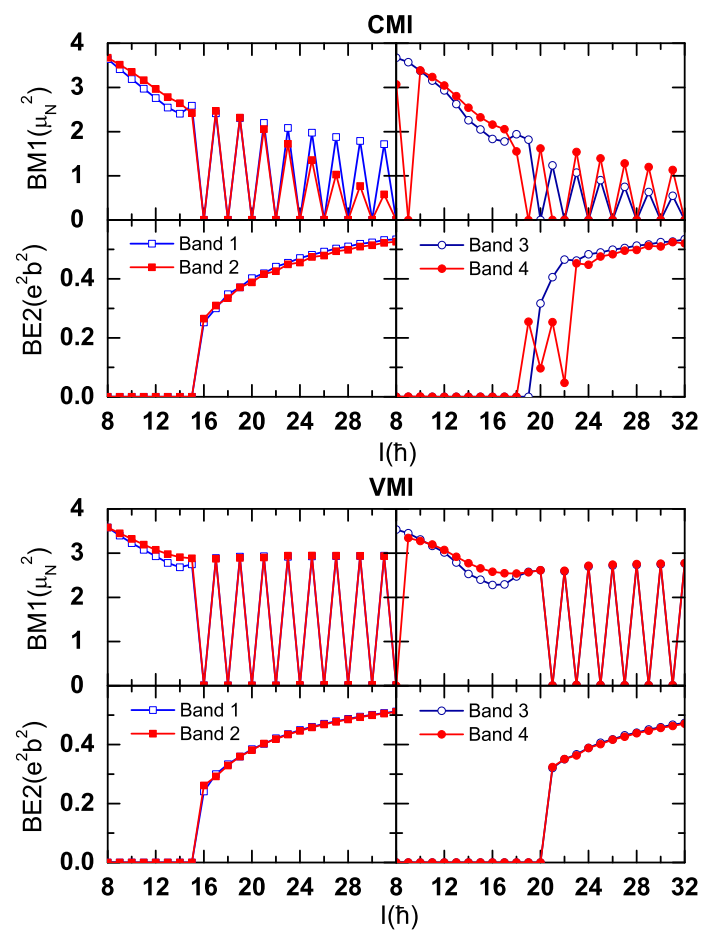

FIG. 2: The intraband electromagnetic transition probabilities $B(M 1)$ and $B(E 2)$ of band 1-4 as functions of the total spin, calculated in PRM with the CMI (upper panel) and VMI (lower panel).

the collective rotation of core becomes dominate with moment of inertia $\mathcal{J} \simeq 3 I \mathrm{MeV}^{-1} \hbar^{2}$.

Besides the small energy difference, the selection rule [10] and the similarity of the electromagnetic transition probabilities are remarkable characters for chiral doublet bands. The intraband electromagnetic transition probabilities $B(M 1 ; I \rightarrow I-1)$ and $B(E 2 ; I \rightarrow I-2)$ of bands 1-4 in the PRM calculations with the CMI and VMI are plotted in Fig. 2. As discussed in Refs. [10, 13], the band 1 and band 2 at $I=15 \hbar$, as well as the band 3 and band 4 at $I=17,18,19 \hbar$, from the calculations with CMI have been exchanged to ensure that the bands are organized based on $B(E 2)$. Similarly, for the calculations with VMI, one should exchange the band 1 and band 2 at $I=17,19,21 \hbar$, as well as the band 3 and band 4 at $I=19,21 \hbar$. It is shown that the intraband electromagnetic transition probabilities $B(M 1)$ and $B(E 2)$ of the band 3 and band 4 are quite similar in the calculations with CMI and almost exactly the same in the calculations with VMI. Moreover, similar behaviors of $B(M 1)$ and $B(E 2)$ as functions of spin in band 3 and band 4 with those in band 1 and band 2 are observed. The obvious difference is the spin value where the odd-even spin staggering in $B(M 1)$ or $B(M 1) / B(E 2)$ occurs. The above phenomena indicate that the static chirality may form between band 3 and band 4 in spin $I \geq 20 \hbar$ region.

It is noted that an evident difference between the cal- 


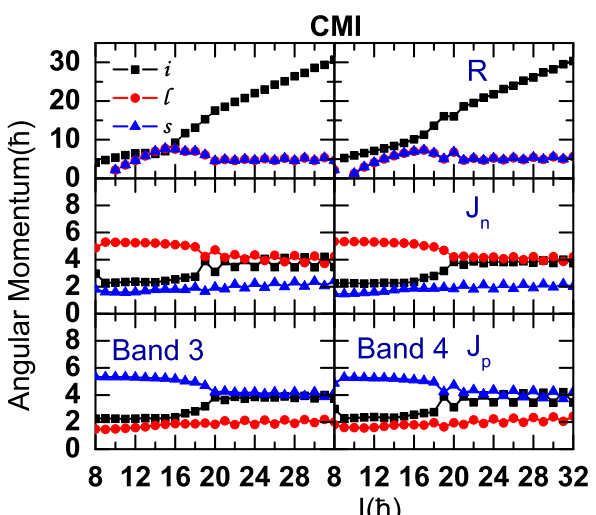

$\mathrm{I}(\hbar)$

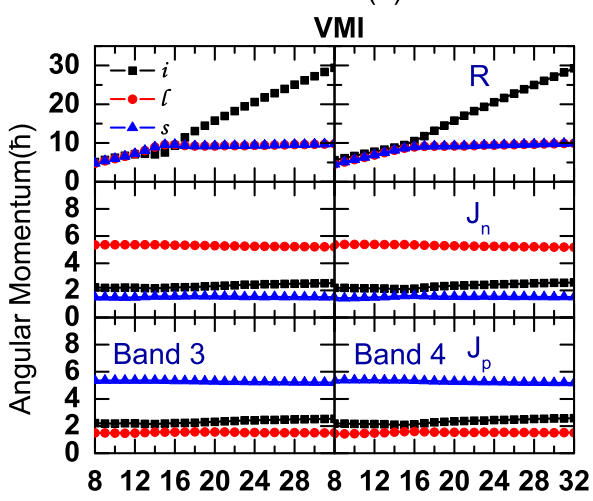

$\mathrm{I}(\hbar)$

FIG. 3: Components along the intermediate (i, squares), short (s, triangles), and long (l, circles) axes of the angular momenta of the core $R_{k}=\left\langle\hat{R}_{k}^{2}\right\rangle^{1 / 2}$, valence neutron $J_{n k}=\left\langle\hat{j}_{n k}^{2}\right\rangle^{1 / 2}$, and valence proton $J_{p k}=\left\langle\hat{j}_{p k}^{2}\right\rangle^{1 / 2}$ for the band 3 and band 4 in PRM with both CMI (upper panel) and VMI (lower panel).

culations with the CMI and VMI is the amplitudes of odd-even spin staggering in $B(M 1)$ values, which are gradually decreasing with the increasing of spin in the calculations of CMI. While, in the calculations of VMI, the amplitudes of odd-even spin staggering are almost spin-independent. It implies that the calculations of VMI would present a little different chiral geometry from that given by the calculations of CMI.

In addition, we have calculated the electromagnetic transition probabilities in band 5 and band 6 . The intraband transitions $B(M 1)$ and $B(E 2)$ of band 5 and band 6 are quite different, irrespective of what $\gamma$ value being used.

The ideal chiral picture in atomic nuclei is formed by three mutually perpendicular angular momenta, i.e., the collective angular momentum of triaxially deformed core with $\gamma=30^{\circ}$ favors alignment along the intermediate axis, whereas the angular momentum vectors of the high$j$ valence particles (holes) favor alignment along the nuclear short (long) axis. Therefore, to examine the chiral geometry of band 3 and band 4 , the rms values of the angular momentum components for the core $R_{k}=\left\langle\hat{R}_{k}^{2}\right\rangle^{1 / 2}$, the valence neutron $J_{n k}=\left\langle\hat{j}_{n k}^{2}\right\rangle^{1 / 2}$, and the valence proton $J_{p k}=\left\langle\hat{j}_{p k}^{2}\right\rangle^{1 / 2}$ for the band 3 and band 4 from the

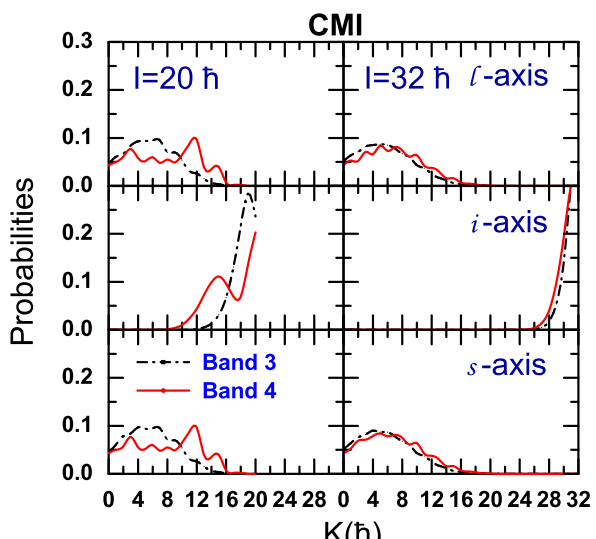

$\mathrm{K}(\hbar)$

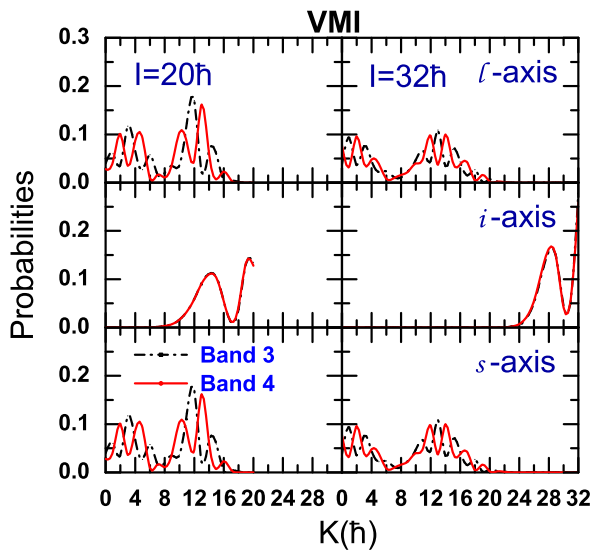

FIG. 4: Probability distributions for projections $K$ of total angular momentum on the long (1), intermediate (i), and short (s) axes for the band 3 and band 4 in PRM with both CMI (upper panel) and VMI (lower panel).

PRM calculations with both CMI and VMI are plotted in Fig. 3, where the indices $k=i, l, s$ represent the intermediate(i), short(s), and long(l) axes respectively.

As shown in the Fig. 3, the calculations with CMI and VMI both present the aplanar rotation for band 3 and band 4 . Specifically, the core angular momentum mainly aligns along the intermediate axis in the calculations with both CMI and VMI due to its largest moment of inertia. For the case of CMI, the $h_{11 / 2}$ valence neutron hole mainly aligns along the long axis at the beginning of the bands. As the total angular momentum $I \geq 20 \hbar$, it lies in the plane defined by the $l$ axis and $i$ axis. In the mean time, the $h_{11 / 2}$ valence proton lies in another plane defined by the $s$ axis and $i$ axis. The three angular momenta together form an aplanar geometry. Such a picture is slightly different from that of VMI, in which case, the $h_{11 / 2}$ valence neutron hole mainly aligns along the $l$ axis, and the valence proton mainly along the $s$ axis at all spin values.

Furthermore, it has been shown in Ref. 17] that the static chiral rotational bands (band 1 and band 2) have similar $K$-distribution, $P_{K}^{I}=\sum_{\alpha}\left|C_{K \alpha}^{I}\right|^{2}$, where the $C_{K \alpha}^{I}$ is the expansion coefficient of total wave function on the 
strong coupling basis [8]. Similar $K$-distribution has been found between static chiral doublet bands. In Fig. 4, we display the $K$-distribution of $I=20 \hbar$ and $32 \hbar$ states in band 3 and band 4 on the $l, i, s$ axes from the PRM calculations with the CMI and VMI. In the calculations with the CMI, the $K$-distribution of $I=20 \hbar$ states is somewhat different in band 3 and band 4 . As the increasing of spin, the $K$-distribution becomes similar, especially at $I=32 \hbar$. In comparison with the results of CMI, the $K$-distribution of band 3 and band 4 is similar even for the state with spin $I=20 \hbar$. It indicates that band 3 and band 4 have the characters of static chirality in spin $I \geq 20 \hbar$ region. Moreover, the calculations with the VMI present better chiral characters in band 3 and band 4 than those in the calculations with the CMI.

In addition, the properties of band 3 and band 4 from the PRM calculations with different triaxiality parameter between $15^{\circ} \leq \gamma \leq 30^{\circ}$ are also examined. We find that the deviation of $\gamma$ from $30^{\circ}$ makes the static chirality violated quickly in band 3 and band 4 . It indicates that the chiral geometry of band 3 and band 4 is much more sensitive to the triaxial deformation than that of band 1 and band 2 .

In summary, the lowest six bands have been studied in the particle-rotor model with the configuration $\pi h_{11 / 2}^{1} \otimes \nu h_{11 / 2}^{-1}$ corresponding to $A \sim 130$ mass region and different values of triaxiality parameter. Both constant and spin-dependent variable moments of inertial have been introduced. The energy spectra, electromagnetic transition probabilities, angular momentum components and $K$-distribution have been examined. All calculated quantities indicate that besides the band 1 and band 2 , the band 3 and band 4 could also form chiral doublet bands in certain spin region $(I \geq 20 \hbar)$ for atomic nuclei with triaxiality parameter $\gamma=30^{\circ}$. However, the chiral geometry of band 3 and band 4 has been found to be strong sensitive to the $\gamma$ value. The calculations with the spin-dependent variable moment of inertial have been shown to present better chiral rotation characters of band 3 and band 4 than that with the constant one.

\section{Acknowledgments}

We thank Professor Jie Meng for useful discussion. This work was partly supported by the Major State 973 Program 2007CB815000 and the National Natural Science Foundation of China under Grant Nos. 10947013, 10975008 and 10775004, the Southwest University Initial Research Foundation Grant to Doctor (No. SWU109011).
[1] S. Frauendorf and J. Meng, Nucl. Phys. A617, 131 (1997).

[2] K. Starosta, T. Koike, C. J. Chiara, et al., Phys. Rev. Lett. 86, 971 (2001).

[3] J. Meng, B. Qi, S. Q. Zhang and S. Y. Wang, Mod. Phys. Lett A23, 2560( 2008).

[4] J. Meng and S. Q. Zhang, J. Phys. G: Nucl. Part. Phys. 37, 064025 (2010).

[5] P. Olbratowski, J. Dobaczewski, J. Dudek, and W. Plociennik, Phys. Rev. Lett. 93, 052501 (2004).

[6] P. Olbratowski, J. Dobaczewski, and J. Dudek, Phys. Rev. C73, 054308 (2006).

[7] V. I. Dimitrov, S. Frauendorf, and F. Dönau, Phys. Rev. Lett. 84, 5732 (2000).

[8] S. Q. Zhang, B. Qi, S. Y. Wang and J. Meng, Phys. Rev. C75, 044307(2007).

[9] J. Peng, J. Meng and S. Q. Zhang, Phys. Rev. C68, 044324 (2003).

[10] T. Koike, K.Starosta, and I. Hamamoto, Phys. Rev. Lett. 93, 172502 (2004).

[11] S. Y. Wang, S. Q. Zhang, B. Qi, and J. Meng, Phys. Rev. C75, 024309 (2007).

[12] T. Koike, K. Starosta, C. J. Chiara, et al., Phys. Rev. C67, 044319 (2003).

[13] B. Qi, S. Q. Zhang, S. Y. Wang, J. M. Yao, and J. Meng, Phys. Rev. C79, 041302 (R) (2009).
[14] S. Y. Wang, S. Q. Zhang, B. Qi, and J. Meng, Chin. Phys. Lett. 24, 664 (2007).

[15] C. M. Petrache, G. B. Hagemann, I. Hamamoto, and K. Starosta, Phys. Rev. Lett. 96, 112502 (2006).

[16] S. Mukhopadhyay, D. Almehed, U. Garg, et al., Phys. Rev. Lett. 99, 172501 (2007).

[17] B. Qi, S. Q. Zhang, J. Meng, S. Y. Wang, and S. Frauendorf, Phys. Lett. B675, 175 (2009).

[18] D. Tonev, et al., Phys. Rev. Lett. 96, 052501 (2006).

[19] D. Tonev, et al., Phys. Rev. C76, 044313 (2007).

[20] J. Meng, J. Peng, S. Q. Zhang, and S. G. Zhou, Phys. Rev. C73, 037303 (2006).

[21] J. M. Yao, B. Qi, S. Q. Zhang, J. Peng, S. Y. Wang, and J. Meng, Phys. Rev. C73, 067302 (2009).

[22] J. Peng, H. Sagawa, S. Q.Zhang, J. M. Yao, Y. Zhang, and J. Meng, Phys. Rev. C77, 024309 (2008).

[23] Ch. Droste, S. G. Rohozinski, K. Starosta, L. Próchniak, and E. Grodner, Eur. Phys. J. A42, 79 (2009)

[24] M. A. J. Mariscotti, G. Scharff-Goldhaber, and B. Buck, Phys. Rev. 178, 1864 (1969).

[25] C. S. Wu and J. Y. Zeng, High Energy Phys. Nucl. Phys. 9, 214 (1985) (in Chinese).

[26] Guo-Jie Chen, Yu-Xin Liu, Hui-Xhao Song, and Hui Cao, Phys. Rev. C73, 034304 (2006). 